\title{
Prevalence of alcohol use among women of reproductive age in Canada
}

\author{
Mélanie Varin, MSc (1); Elia Palladino, MSc (1,2); Kate Hill MacEachern, PhD (1); Lisa Belzak, MHSc (1); \\ Melissa M. Baker, PhD (1)
}

Tweet this article

\begin{abstract}
Introduction: Reporting on alcohol use among women of reproductive age in Canada addresses a major gap in evidence.

Methods: We assessed the prevalence of weekly and heavy alcohol consumption among women aged 15 to 54 years by sociodemographic characteristics, province of residence and concurrent use of other substance(s) using data from the 2019 Canadian Community Health Survey.

Results: Of the target population, $30.5 \%$ reported weekly and $18.3 \%$ reported heavy alcohol consumption in the past year. Prevalence varied by sociodemographic characteristics, province and substance use. The most notable and significant differences were to do with cannabis use and smoking.

Conclusion: This information can guide health care providers in assessing alcohol consumption and in promoting low-risk alcohol drinking to prevent alcohol exposure during pregnancy.
\end{abstract}

Keywords: substance use, polysubstance use, women of reproductive age, Canada's LowRisk Alcohol Drinking Guidelines

\section{Introduction}

Alcohol is widely used in Canada, with over $75 \%$ of the population aged 15 years and over reporting alcohol consumption in the previous year. ${ }^{1}$ Numerous adverse outcomes are associated with alcohol consumption, commonly referred to as alcohol-related harms. These include, but are not limited to, physical injury ${ }^{2}$, adverse physical and mental health effects ${ }^{2,3}$, cancer $^{4}$, cirrhosis of the liver ${ }^{5}$ and even death. . $, 6,7^{-}$

Canada's Low-Risk Alcohol Drinking Guidelines were published in 2011 to raise awareness to help reduce alcohol-related harms. ${ }^{8}$ These guidelines outline best practices for safe alcohol consumption, including recommendations to abstain from alcohol when planning to become pregnant or when pregnant. ${ }^{8}$ Harm reduction strategies help clinicians and public health professionals promote low-risk alcohol consumption in subpopulations who would benefit from increased awareness of the health risks associated with drinking behaviours. This is particularly important for women of reproductive age as they may have a planned or unplanned pregnancy during this time in their lives, and alcohol consumption while pregnant could have significant impacts on the fetus. In 2017, $4.2 \%$ of women in Canada who had given birth in the last 5 years reported consuming any alcohol during their pregnancy. ${ }^{9}$

In this study, we (1) report on the prevalence of weekly and heavy alcohol

\section{Highlights}

- In $2019,30.5 \%$ of women of reproductive age reported weekly alcohol consumption.

- In $2019,18.3 \%$ of women of reproductive age reported heavy alcohol consumption that exceeds Canada's Low-Risk Alcohol Drinking Guidelines.

- These proportions varied by sociodemographic characteristics, province of residence, smoking status and cannabis use.

- Prevalence estimates for weekly and heavy alcohol consumption were between 2 and 3 times higher for women who reported being current, former or experimental tobacco smokers or who had reported consuming cannabis in the past year.

consumption among women aged 15 to 54 years old in Canada; and (2) explore alcohol consumption in this population by sociodemographic characteristics, province of residence, smoking status and cannabis use. To our knowledge, there are no historic or current national estimates that report on alcohol consumption by women of reproductive age. We aim to fill that gap in this paper.

\section{Methods}

Using data from the Canadian Community Health Survey (CCHS) annual cycle for 2019 (January to December), we assessed two alcohol consumption behaviours in the population in Canada who selfreported as female and who were between 
15 and 54 years old: weekly alcohol consumption and heavy alcohol consumption. The CCHS is a national cross-sectional survey conducted by Statistics Canada that provides health information. The 2019 CCHS coverage excluded women living in the territories, on First Nation reserves or other Indigenous settlements in the provinces, as well as full-time members of the Canadian Armed Forces and individuals in institutions.

Further detail about the CCHS design and sampling framework can be found on the Statistics Canada website. ${ }^{1}$

\section{Weekly alcohol consumption}

Respondents were asked, "During the past 12 months, how often did you drink alcoholic beverages?" Response options included "less than once a month," "once a month," "2 to 3 times a month," "once a week," "2 to 3 times a week," "4 to 6 times a week" or "every day." Weekly alcohol consumption was defined as responses of "once a week," "2 to 3 times a week," " 4 to 6 times a week" or "every day." We excluded from the analysis women of reproductive age who responded with "don't know," "refusal" or "not stated" (n = 72).

\section{Heavy alcohol consumption}

Respondents were asked, "How often in the past 12 months have you had four or more drinks on one occasion?" Response options were "never," "less than once a month," "once a month," "2 to 3 times a month," "once a week" or "more than once a week." Heavy alcohol consumption was defined as responses of "once a month," "2 to 3 times a month," "once a week" or "more than once a week." 10 Women of reproductive age who responded with "don't know," "refusal" or "not stated" were excluded from the analysis $(\mathrm{n}=108)$.

We estimated the weighted prevalence (with 95\% confidence interval) of weekly and heavy alcohol consumption of the target population. We used as numerator the total number of women of reproductive age who reported weekly or heavy alcohol consumption and as the denominator the total number of women of reproductive age who responded to the question.

We disaggregated estimates by age group, household income quintile, province of residence, marital status, urban/rural status, immigrant status, employment in past 12 months, current student status, cannabis use and tobacco smoking status in the last year. Weighted prevalence estimates were calculated with survey sampling weights provided by Statistics Canada. Variance was estimated using the bootstrap method, and SAS Enterprise Guide version 7.1 (SAS Institute Inc., Cary, NC, USA) was used for statistical analyses. Statistical significance was established by examining overlapping confidence intervals.

\section{Results}

Overall, $30.5 \%$ of women of reproductive age reported consuming alcohol weekly in the past year, and $18.3 \%$ reported engaging in heavy alcohol consumption. These prevalence estimates varied after disaggregation (Table 1). Statistically significant differences are detailed in the following sections.

\section{Weekly alcohol consumption}

Rates of weekly alcohol consumption ("regular use") increased with age (from $7.1 \%$ to $35.6 \%$ ) and income ( $19.8 \%$ to $41.2 \%$ ). The highest prevalence of weekly alcohol consumption in this target population was in Quebec (41.0 \%) and the lowest (20.0\%) in New Brunswick. A higher proportion of women of reproductive age who were married or living common-law reported weekly alcohol consumption (33.7\%) than those who were widowed, separated, divorced, single or never married $(26.3 \%)$.

Non-immigrants (37.6\%) and non-students $(33.4 \%)$ had a higher prevalence of weekly alcohol consumption than immigrants (13.6\%) and students (19.1\%). The percentage of women of reproductive age who worked at a job or business in the past year reporting weekly alcohol consumption was higher (33.9\%) than for those who did not work at a job or business $(13.9 \%)$.

Prevalence rates of weekly alcohol use were approximately 2 times higher for current $(40.3 \%)$, former $(47.0 \%)$ and experimental $(43.2 \%)$ tobacco smokers than for lifetime abstainers (21.6\%). Women of reproductive age who reported using cannabis in the past 12 months reported weekly alcohol use $(46.0 \%)$ that was almost twice that of those who did not use cannabis in the past 12 months $(26.3 \%)$.

\section{Heavy alcohol consumption}

Women aged 20 to 29 years had the highest prevalence of heavy alcohol consumption $(25.2 \%)$. Rates of heavy alcohol consumption increased with income (from $15.4 \%$ to $23.7 \%$ ). The highest prevalence of heavy alcohol consumption among women of reproductive age was in Newfoundland and Labrador $(26.6 \%)$ and the lowest in New Brunswick (14.6\%). A higher percentage of widowed, separated, divorced, single or never married women of reproductive age $(21.1 \%)$ reported heavy alcohol consumption than those who were married or living common-law (16.3\%).

The percentage of women of reproductive age reporting heavy alcohol consumption was higher for those living in a rural area $(22.4 \%)$ than for those in urban areas $(17.6 \%)$. A higher proportion who were non-immigrants $(23.1 \%)$ and who worked at a paid job in the past 12 months $(20.4 \%)$ reported heavy drinking compared to immigrants $(6.9 \%)$ and to those who did not work a paid job in the last year $(8.2 \%)$.

Current (35.4\%), former $(27.3 \%)$ and experimental $(23.0 \%)$ tobacco smokers had proportions of heavy alcohol consumption that were between 2 and 3 times higher than lifetime abstainers $(11.3 \%)$. Women of reproductive age who reported consuming cannabis in the past 12 months (39.1\%) had a rate of heavy alcohol consumption that was over 3 times higher than women of reproductive age who did not consume cannabis in the past 12 months $(12.4 \%)$.

\section{Discussion}

In $2019,30.5 \%$ of Canadian women of reproductive age reported consuming alcohol weekly and $18.3 \%$ reported engaging in heavy alcohol consumption. Although there are slight differences in the definitions of heavy (having four or more drinks on one occasion at least once a month in the past year) and binge drinking (having four or more drinks on one occasion at least once in the past year), the proportion of heavy alcohol consumption is similar to the 2019 median prevalence (19.2\%) of binge drinking among women aged 18 to 44 years in the United States. ${ }^{11}$

We found that the proportion of weekly and heavy alcohol consumption was highest among women of reproductive age in 
TABLE 1

Prevalence estimates for weekly and heavy consumption of alcohol in the last year among women of reproductive age, by sociodemographic and other characteristics, Canada, provinces, 2019

\begin{tabular}{|c|c|c|c|}
\hline \multirow[b]{2}{*}{ Variables } & \multicolumn{3}{|c|}{ Prevalence $(95 \% \mathrm{CI})$} \\
\hline & $\%$ in sample & $\begin{array}{l}\text { \% who reported weekly } \\
\text { alcohol consumption }\end{array}$ & $\begin{array}{c}\% \text { who reported heavy alcohol consumption } \\
\text { once a month or more frequently }\end{array}$ \\
\hline \multicolumn{4}{|l|}{ Age group, years } \\
\hline $15-19$ & $9.8(9.1,10.6)$ & $7.1(5.1,9.0)$ & $10.6(8.1,13.1)$ \\
\hline $20-29$ & $23.9(22.9,24.9)$ & $29.8(27.1,32.5)$ & $25.2(22.8,27.7)$ \\
\hline $30-39$ & $27.8(26.7,29.0)$ & $32.4(30.1,34.6)$ & $16.7(15.0,18.4)$ \\
\hline $40-54$ & $38.5(37.6,39.3)$ & $35.6(33.6,37.5)$ & $17.2(15.7,18.7)$ \\
\hline \multicolumn{4}{|l|}{ Income } \\
\hline Q1 (lowest income) & $21.8(20.7,23.0)$ & $19.8(17.3,22.3)$ & $15.4(13.1,17.7)$ \\
\hline Q2 & $19.2(18.1,20.3)$ & $27.1(24.3,29.8)$ & $17.3(15.1,19.6)$ \\
\hline Q3 & $20.1(19.0,21.3)$ & $30.6(27.9,33.2)$ & $16.5(14.4,18.5)$ \\
\hline Q4 & $20.0(18.9,21.1)$ & $35.6(32.7,38.6)$ & $19.3(17.0,21.6)$ \\
\hline Q5 (highest income) & $18.8(17.8,19.8)$ & $41.2(38.3,44.1)$ & $23.7(21.1,26.3)$ \\
\hline \multicolumn{4}{|l|}{ Province of residence } \\
\hline British Columbia & $12.9(12.7,13.2)$ & $29.9(26.6,33.2)$ & $20.5(17.5,23.4)$ \\
\hline Alberta & $12.4(12.1,12.6)$ & $31.8(28.4,35.3)$ & $18.0(15.2,20.9)$ \\
\hline Saskatchewan & $2.9(2.8,3.0)$ & $27.9(22.3,33.6)$ & $17.1(12.6,21.5)$ \\
\hline Manitoba & $3.5(3.4,3.6)$ & $30.1(24.8,35.4)$ & $20.4(15.6,25.2)$ \\
\hline Ontario & $40.6(40.1,41.0)$ & $25.5(23.6,27.5)$ & $15.5(13.8,17.2)$ \\
\hline Quebec & $21.7(21.4,22.1)$ & $41.0(38.3,43.7)$ & $21.6(19.4,23.8)$ \\
\hline New Brunswick & $1.9(1.8,1.9)$ & $20.0(14.8,25.3)$ & $14.6^{\mathrm{C}}(10.1,19.1)$ \\
\hline Nova Scotia & $2.5(2.4,2.6)$ & $34.1(29.0,39.2)$ & $23.1(18.4,27.8)$ \\
\hline Prince Edward Island & $0.4(0.4,0.4)$ & $23.9^{\mathrm{C}}(16.7,31.1)$ & $19.4^{\mathrm{C}}(13.2,25.6)$ \\
\hline Newfoundland and Labrador & $1.2(1.2,1.3)$ & $21.3(15.0,27.5)$ & $26.6(20.2,32.9)$ \\
\hline \multicolumn{4}{|l|}{ Marital status } \\
\hline Married/living common-law & $57.4(56.1,58.6)$ & $33.7(32.0,35.3)$ & $16.3(15.0,17.5)$ \\
\hline Widowed, separated, divorced, single, never married & $42.6(41.4,43.9)$ & $26.3(24.5,28.1)$ & $21.1(19.4,22.7)$ \\
\hline \multicolumn{4}{|l|}{ Urban/rural area of residence } \\
\hline Urban & $85.5(84.7,86.2)$ & $30.1(28.7,31.4)$ & $17.6(16.5,18.7)$ \\
\hline Rural & $14.5(13.8,15.3)$ & $33.0(30.6,35.5)$ & $22.4(20.3,24.6)$ \\
\hline \multicolumn{4}{|l|}{ Immigrant status } \\
\hline Yes & $29.8(28.4,31.1)$ & $13.6(11.9,15.3)$ & $6.9(5.7,8.2)$ \\
\hline No & $70.2(68.9,71.6)$ & $37.6(36.2,39.0)$ & $23.1(21.8,24.3)$ \\
\hline \multicolumn{4}{|l|}{ Worked at a paid job or business in past 12 months } \\
\hline Yes & $83.2(82.2,84.3)$ & $33.9(32.6,35.3)$ & $20.4(19.2,21.5)$ \\
\hline No & $16.8(15.7,17.8)$ & $13.9(11.7,16.2)$ & $8.2(6.5,9.9)$ \\
\hline \multicolumn{4}{|l|}{ Current student } \\
\hline Yes & $20.2(19.2,21.3)$ & $19.1(16.7,21.5)$ & $16.6(14.3,18.8)$ \\
\hline No & $79.8(78.7,80.8)$ & $33.4(32.0,34.8)$ & $18.8(17.6,19.9)$ \\
\hline \multicolumn{4}{|l|}{ Smoking status } \\
\hline Lifetime abstainer & $59.4(58.0,60.7)$ & $21.6(20.2,23.1)$ & $11.3(10.2,12.4)$ \\
\hline Current smoker & $13.4(12.6,14.2)$ & $40.3(37.2,43.4)$ & $35.4(32.3,38.5)$ \\
\hline Former smoker & $14.0(13.1,14.9)$ & $47.0(43.9,50.2)$ & $27.3(24.5,30.0)$ \\
\hline Experimental smoker & $13.2(12.3,14.2)$ & $43.2(39.4,46.9)$ & $23.0(19.9,26.2)$ \\
\hline
\end{tabular}


TABLE 1 (continued)

Prevalence estimates for weekly and heavy consumption of alcohol in the last year among women of reproductive age, by sociodemographic and other characteristics, Canada, provinces, 2019

\begin{tabular}{|c|c|c|c|}
\hline \multirow[b]{2}{*}{ Variables } & \multicolumn{3}{|c|}{ Prevalence $(95 \% \mathrm{CI})$} \\
\hline & $\%$ in sample & $\begin{array}{l}\% \text { who reported weekly } \\
\text { alcohol consumption }\end{array}$ & $\begin{array}{l}\% \text { who reported heavy alcohol consumption } \\
\text { once a month or more frequently }\end{array}$ \\
\hline \multicolumn{4}{|l|}{ Cannabis use in past 12 months } \\
\hline Yes & $23.0(21.9,24.2)$ & $46.0(43.2,48.7)$ & $39.1(36.4,41.8)$ \\
\hline No & $77.0(75.8,78.1)$ & $26.3(25.0,27.6)$ & $12.4(11.5,13.4)$ \\
\hline Overall & - & $30.5(29.3,31.7)$ & $18.3(17.3,19.3)$ \\
\hline
\end{tabular}

Data source: Canadian Community Health Survey 2019.

Abbreviation: $\mathrm{Cl}$, confidence interval.

c As per the Statistics Canada's sampling variability guidelines, prevalence estimates should be interpreted with caution, as the coefficient of variation is between $15.1 \%$ and $25.0 \%$.

the highest income quintile, who were not immigrants, who worked at a job in the past 12 months and who were not students. We also found heterogeneity between self-reported alcohol consumption behaviours. For example, although women of reproductive age who were married or in a common-law relationship had a higher prevalence of weekly alcohol consumption than those who were widowed, separated, divorced, single or had never married, this pattern was the opposite for heavy alcohol consumption behaviour. Similarly, prevalence of weekly alcohol consumption was highest for those in the 40-49-year age group, whereas prevalence for heavy alcohol consumption was highest for those in the 20-29-year age group. Based on these differences, future studies should examine more than one consumption behaviour across various social determinants of health to have a better understanding of alcohol use in this population.

Our most notable findings were to do with smoking and cannabis use, which may be indicative of polysubstance use. Prevalence of past year weekly and heavy alcohol consumption was 2 to 3 times higher for women of reproductive age who were current, former or experimental tobacco smokers or who had reported consuming cannabis in the past year. This information can help health care providers assess polysubstance use in this population and promote low-risk alcohol drinking as a measure to prevent exposure to alcohol during pregnancy.

\section{Strength and limitations}

Our study was the first to provide nationally representative prevalence estimates of two alcohol use behaviours among women of reproductive age and to disaggregate by various sociodemographic and substance use variables. While our findings address a gap in evidence, we have a few limitations to highlight.

First, as the data are cross-sectional we cannot establish temporality. Second, all of the data in the analysis were selfreported and therefore subject to reporting and social desirability bias. Lastly, due to the CCHS's exclusion criteria, we were unable to include information from women of reproductive age living in the territories or on First Nation reserves.

\section{Further research}

To identify groups of women of reproductive age who may benefit from increased awareness of low-risk alcohol-drinking guidelines, future research should examine associations between sociodemographic characteristics, consumption of other substances and alcohol use.

\section{Acknowledgements}

The authors would like to thank our colleagues in the Maternal, Child and Youth Health Division for extending the invitation to collaborate and jointly submit At-a-glance articles. We would also like to thank Adam Probert, Claudia Lagacé and Sarah Palmeter for assisting with interpretation of the results and for revising this At-a-glance article.

\section{Conflicts of interest}

None.

\section{Authors' contributions and statement}

All authors advised on the conception and design of the secondary data analysis. MV drafted this At-a-glance article and conducted the descriptive analysis. All coauthors interpreted the data and critically revised and provided feedback on every draft of this At-a-glance article.

The content and views expressed in this At-a-glance article are those of the authors and do not necessarily reflect those of the Government of Canada.

\section{References}

1. Statistics Canada. Canadian Community Health Survey - Annual Component (CCHS): Detailed information for 2019 [Internet]. Ottawa (ON): Statistics Canada; [modified 2019 Nov 18; cited 2020 Nov 16]. Available from: https:// www23.statcan.gc.ca/imdb/p2SV.pl ?Function $=$ getSurvey $\& I d=1208978$

2. Shield KD, Kehoe T, Taylor B, Patra J, Rehm J. Alcohol-attributable burden of disease and injury in Canada, 2004. Int J Public Health. 2012;57(2):391401. https://doi.org/10.1007/s00038 $-011-0247-7$

3. Iranpour A, Nakhaee N. A review of alcohol-related harms: a recent update. Addict Health. 2019 Apr;11(2):129-137. https://doi.org/10.22122/ahj.v11i2.225

4. The Lancet. Alcohol and cancer. Lancet. 2017;390(10109):2215. https://doi.org /10.1016/S0140-6736(17)32868-4

5. Roerecke M, Vafaei A, Hasan OS, et al. Alcohol consumption and risk of liver cirrhosis: a systematic review and meta-analysis. Am J Gastroenterol. 2019;114(10):1574-86. https://doi.org /10.14309/ajg.0000000000000340 
6. Orpana H, Giesbrecht N, Hajee A, Kaplan MS. Alcohol and other drugs in suicide in Canada: opportunities to support prevention through enhanced monitoring. Inj Prev. 2021;27(2):194200. https://doi.org/10.1136/injuryprev $-2019-043504$

7. Ramstedt M. Alcohol consumption and alcohol-related mortality in Canada, 1950-2000. Can J Public Health. 2004; 95(2):121-6. https://doi.org/10.1007 /BF03405779

8. National Alcohol Strategy Advisory Committee. Canada's Low-Risk Alcohol Drinking Guidelines. Ottawa (ON): Canadian Centre on Substance Use and Addiction; 2018.

9. Centre for Surveillance and Applied Research. Perinatal Health Indicators (PHI) Data Tool. Public Health Infobase [Internet]. Ottawa (ON): Public Health Agency of Canada; [modified 2020 Oct 20; cited 2021 April 16]. Available from: https://health-infobase.canada .ca/phi/data-tool/index? Dom $=1$

10. Statistics Canada. Health fact sheets: Heavy drinking, 2018 [Internet]. Ottawa (ON): Statistics Canada; [modified 2019 Jun 25; cited 2020 Nov 16]. Available from: https://www150.statcan.gc.ca /n1/pub/82-625-x/2019001/article /00007-eng.htm

11. National Center on Birth Defects and Developmental Disabilities. Fetal alcohol spectrum disorders (FASDs): Statelevel estimates of alcohol use among women - 2019 [Internet]. Atlanta (GA): Centers for Disease Control and Prevention; [reviewed 2021 May 14; cited 2021 May 27]. Available from: https://www.cdc.gov/ncbddd/fasd /data-maps-2019.html 